\title{
Determining Building Interior Structures using Compressive Sensing
}

\author{
Eva Lagunas, Moeness G. Amin, Fauzia Ahmad, and Montse Nájar
}

\begin{abstract}
We consider imaging of the building interior structures using Compressive Sensing (CS) with applications to through-the-wall imaging and urban sensing. We consider a monostatic synthetic aperture radar imaging system employing stepped frequency waveform. The proposed approach exploits prior information of building construction practices to form an appropriate sparse representation of the building interior layout. We devise a dictionary of possible wall locations, which is consistent with the fact that interior walls are typically parallel or perpendicular to the front wall. The dictionary accounts for the dominant normal angle reflections from exterior and interior walls for the monostatic imaging system. Compressive sensing is applied to a reduced set of observations to recover the true positions of the walls. Additional information about interior walls can be obtained using a dictionary of possible corner reflectors, which is the response of the junction of two walls. Supporting results based on simulation and laboratory experiments are provided. It is shown that the proposed sparsifying basis outperforms the conventional through-the-wall CS model, the wavelet sparsifying basis, and the block sparse model for building interior layout detection.
\end{abstract}

\section{Index Terms}

Through-the-Wall Radar Imaging, Compressive Sensing, Building layout.

The work by M. G. Amin and F. Ahmad was supported in part by ONR under grant N00014-11-1-0576 and in part by ARO and ARL under contract W911NF-11-1-0536. The work by E. Lagunas was performed during her tenure as a Visiting Research Associate at the Center for Advanced Communications, Villanova University, USA. E. Lagunas and M. Nájar are partially supported by the Spanish Ministry of Science and Innovation (Ministerio de Ciencia e Innovación) under project TEC2011-29006-C03-02 (GRE3N-LINK-MAC).

E. Lagunas and M. Nájar are with the Signal Theory and Communications Department, Universitat Politècnica de Catalunya, Barcelona, 08034 Spain. E-mail: \{eva.lagunas,montse.najar\}@upc.edu.

M.G. Amin and F. Ahmad are with the Radar Imaging Lab, Center for Advanced Communications, Villanova University, Villanova, PA 19085, USA. E-mail: \{moeness.amin,fauzia.ahmad\}@villanova.edu. 


\section{INTRODUCTION}

Through-the-Wall Radar Imaging (TWRI) is an emerging technology that allows imaging of building interiors through exterior walls [1]-[9]. TWRI combines electromagnetic waves transmitted and received at several different locations along an array aperture, whether physical or synthetic, to obtain a two-dimensional (2D) or three-dimensional (3D) image of the region of interest behind the front wall. This type of technology is of great interest in numerous civilian and military applications, including search-and-rescue missions [10].

High resolution imaging is associated with large bandwidth signals and long array apertures. However, this demands acquisition and processing of large amounts of data. In order to expedite data acquisition and alleviate processing bottlenecks, Compressive Sensing (CS) has emerged as an effective approach that allows data compression while sensing [11]-[14]. Besides the goal of achieving fast data acquisition, using few measurements to generate a high-resolution image in TWRI is important logistically, as some of the data observations in time, space and frequency can be difficult or impossible to attain. Such difficulty may be attributed to interference within a frequency band or at specific frequencies, and also due to the inability of the EM waves to reach the behind-the-wall scene from certain aspect angles.

In this paper, we address the problem of imaging building interior structures using a reduced number of measurements. The conventional backprojection method is commonly adopted in TWRI for image formation. It has been shown that missing a large number of data samples compromises the backprojection image quality and impedes detection of indoor targets [15]. In this paper, we consider interior walls as targets and attempt to reveal the building layout based on $\mathrm{CS}$ and sparse image reconstruction techniques. Construction practices suggest the exterior and interior walls to be parallel or perpendicular to each other. This enables sparse representations of the scene using dictionaries of possible wall and corner orientations and locations. Conventional CS recovery algorithms can then be applied to a reduced number of observations to recover the positions of various walls, which is a primary goal in through-the-wall sensing.

Detection of building interior structures assuming availability of full data measurements was presented in [16]-[21]. In [16], the problem of building structure estimation was solved using microwave ray tomography. The authors developed a Bayesian formulation and used Markov chain Monte Carlo procedure to sample the posterior distribution. In [17], an iterative proce- 
dure based on the jump-diffusion algorithm was proposed to estimate the building layout for moving sensor systems. The building layout mapping technique discussed in [18] relates the measurements to a list of floor-plan topologies through a graph-theoretical approach. Direct interpretation of the radar image was proposed in [19], where an image of the full building layout and the interior targets was obtained by combining images obtained from two sides of the building. With proper angular radar illuminations, which certainly avoid the front wall returns, the corner features created by the junction of walls of a room are preserved. This idea was exploited in [20], [21], where a building feature based approach was applied to estimate the type and location of different canonical scattering mechanisms. However, the oblique scan images must be clear so that all corners can be identified by a simple search of local maxima, which is not a straightforward assumption when a reduced number of data samples is considered.

In this paper, the building mapping problem is solved using fewer samples, acquired by a ground-based synthetic aperture radar (SAR) system that is located parallel to the front wall. We consider a two-dimensional (2D) scene model and devise a dictionary of possible wall locations for sparse representation, which is consistent with the interior walls being parallel or perpendicular to the front wall. A follow on step is to use a dictionary of possible corner reflectors, which is the type of reflection caused by the junction of walls that are perpendicular to each other. Determining corner reflectors along the wall segments can indicate the wall extent. Similar approaches, but with different models and formulations, were recently proposed in [22], [23]. In [22], the Hough Transform (HT) domain for continuous infinite-length line detection was strictly discretized for tunnel detection in Ground Penetrating Radar (GPR). The TWRI problem, considered in [23], provides an improvement to the conventional HT reconstruction assuming knowledge of the orientation of walls and by applying sharp windowing to the resulting HT. Reference [23], however, did not deal with CS and reduced data volume.

The proposed approach provides reliable determination of building interior layout while achieving substantial reduction in data volume. Using simulated data as well as real data collected in a laboratory setting, we demonstrate the effectiveness of the proposed sparsifying dictionary for reconstruction of building structures. In particular, we show that the proposed approach outperforms the conventional point target based through-the-wall CS model, the wavelet sparsifying basis, and the block sparse model for imaging of building structures.

The remainder of the paper is organized as follows. Section II introduces TWRI and presents 
the conventional image formation method. Section III reviews the compressive sensing theory applied to TWRI. Section IV describes different image sparsifying basis including the proposed one. Section V discusses the multipath and reverberation effects on the building layout detection problem and Section VI provides supporting results based on numerical and experimental data. Finally, conclusions are drawn in Section VII.

Throughout the paper, we denote vectors by boldface lowercase letters and matrices by boldface uppercase letters. For a given matrix $\mathbf{A}, \mathbf{A}^{T}$ and $\mathbf{A}^{H}$ denotes its transpose and conjugate transpose, respectively. $\mathbf{I}_{Q}$ indicates an identity matrix of dimension $Q \times Q$. Function $\operatorname{sinc}(x)$ stands for $\sin (x) / x$. We use the positive sign convention for time harmonic variables, i.e. $\exp (+j \omega t)$, where $\omega$ is the radian frequency.

\section{TWRI Signal Model And CONVEntional IMAge Formation}

Consider a SAR system in which a single antenna at one location transmits and receives the wideband radar signal, then moves to the next location, and repeats the same operation along the $x$-axis parallel to the front wall, as shown in Fig. 1. Assume $N$ antenna locations, which can be uniformly or randomly spaced. Since it is common practice to build walls either parallel or perpendicular to each other, all interior walls present in the scene are assumed to be parallel or perpendicular to the array. Let the antenna at the $n$th location illuminate the scene with a stepped-frequency signal of $M$ frequencies, which are equispaced over the desired bandwidth $\omega_{M-1}-\omega_{0}$,

$$
\omega_{m}=\omega_{0}+m \Delta_{\omega}, \quad m=0, \ldots, M-1
$$

where $\omega_{0}$ and $\Delta_{\omega}$ denote the lowest frequency in the bandwidth spanned by the stepped-frequency signal and the frequency step size, respectively. Note that, due to the specular nature of the wall reflections, contributions from interior building walls only parallel to the antenna array will mostly be received at the array. Detection of interior perpendicular walls would only be possible when imaging from another side of the building. Detection of corner reflectors along the interior parallel walls could also reveal the wall junctions. For a scene consisting of $P$ point targets, $I_{w}$ interior walls parallel to the array axis and $C$ corner reflectors, the signal received by the $n$-th transceiver at the $m$-th frequency can be expressed as,

$$
y(m, n)=A_{w} e^{-j \omega_{m} \tau_{w}}+\sum_{i=0}^{I_{w}-1} A_{w_{i}} e^{-j \omega_{m} \tau_{w_{i}}}+\sum_{p=0}^{P-1} A_{p} e^{-j \omega_{m} \tau_{p, n}}+\sum_{q=0}^{C-1} \Upsilon_{[q, n]} A_{q} S_{m, q, n} e^{-j \omega_{m} \tau_{q, n}}
$$


with

$$
S_{m, q, n}=\operatorname{sinc}\left(\omega_{m} \frac{L_{q}}{c} \sin \left(\theta_{q, n}-\bar{\theta}_{q}\right)\right)
$$

The function $\Upsilon_{[q, n]}$ works as an indicator function in the following way,

$$
\Upsilon_{[q, n]}= \begin{cases}1 & \text { if } n \text {-th antenna illuminates the concave side of the } q \text {-th corner } \\ 0 & \text { otherwise }\end{cases}
$$

That is, wall corners will only be revealed if the scanning path is on the concave side of the corner. In eqn. (2), $A_{w}, A_{w_{i}}, A_{p}$ and $A_{q}$ contain the amplitude of each scatterer. In general, each amplitude has three components: the attenuation caused by the distance between the scatterer and the receiver, the attenuation caused by the propagation through the front wall, and the complex reflectivity of each scatterer. The variables $L_{q}$ and $\bar{\theta}_{q}$, respectively, define the length and the orientation angle of the $q$-th corner reflector, whereas $\theta_{q, n}$ is the aspect angle associated with the $q$-th corner reflector and the $n$-th antenna. It is noted that the wall and target responses are assumed to be independent of frequency and aspect angle. In the above equation, $\tau_{w}, \tau_{w_{i}}, \tau_{p, n}$ and $\tau_{q, n}$, respectively, represent the two-way traveling time of the signal from the $n$-th antenna to the wall, from the $n$-th antenna to the $i$-th interior wall, between the $n$-th antenna and the $p$-th target, and between the $n$-th antenna and the $q$-th corner. As the wall is a specular reflector and the antennas are located parallel to the front wall, the delays $\tau_{w}$ and $\tau_{w_{i}}$ are independent of the variable $n$, as evident in the subscripts.

A radar image is generated from the $M N$ observations, $y(m, n), m=0, \ldots, M-1, n=$ $0, \ldots, N-1$, using a wideband synthetic aperture beamformer as follows. The scene being imaged is partitioned into a finite number of pixels, $N_{x} \times N_{z}$, in crossrange and downrange. That is, the scene can be represented by the complex reflectivity function $r(k, l), k=0, \ldots, N_{x}-1, l=$ $0, \ldots, N_{z}-1$. The complex composite signal, corresponding to the $(k, l)$ th pixel, is obtained by applying a set of focusing delays, $\tau_{(k, l), n}$, to align all signal returns from the $(k, l)$ th pixel, and then summing the results [24],

$$
r(k, l)=\frac{1}{M N} \sum_{n=0}^{N-1} \sum_{m=0}^{M-1} y(m, n) e^{j \omega_{m} \tau_{(k, l), n}} .
$$

Note that the focusing delay, $\tau_{(k, l), n}$ corresponds to the two-way signal propagation time between the $n$th antenna location and the $(k, l)$ th pixel. The process described by eqn. (5) is performed for all $N_{x} \times N_{z}$ pixels to generate the image of the scene. 
The aforementioned image formation procedure is commonly known as frequency-domain backprojection. It assumes the point target model and does not take into account the specular nature of the wall reflections, particularly the normal angle radar returns from the wall when the antennas are located parallel to the wall.

\section{COMPRESSIVE SENSING FOR TWRI}

The CS theory [11], [12], [25] states that good accuracy reconstruction of a signal can be achieved with signal sub-sampling, provided that the following requirements are satisfied: (1) the signal to be recovered must be sparse in an appropriate basis, and (2) the signal should have a linear relationship with the sampled data measurements. CS framework has been shown to be useful for reducing data acquisition time and alleviating processing bottlenecks in TWRI [26]-[30].

In CS-based TWRI, the scene itself is usually considered sparse for CS application [26], [29]. This sparsity-driven approach operates under the assumption of the point target model and applies the sparsity condition directly to the scene, arguing that the number of point targets $P$ present is typically much smaller than the number of scene pixels. That is, $P<<N_{x} \times N_{z}$. Let

$\mathbf{y}_{n}=\left[\begin{array}{llll}y(0, n) & y(1, n) & \ldots & y(M-1, n)\end{array}\right]^{T}$ represent the received signal vector corresponding to the $M$ frequencies at the $n$-th antenna location, and $\mathbf{r}$ be the concatenated $N_{x} N_{z} \times 1$ scene reflectivity vector corresponding to the spatial sampling grid. Then, under the point target model and using eqn. (2), we obtain the relationship between the measurements at the $n$th antenna location and the scene reflectivity as

$$
\mathbf{y}_{n}=\Psi_{n} \mathbf{r}
$$

where $\Psi_{n}$ is an $M \times N_{x} N_{z}$ matrix, whose rows are given by,

$$
\left[\boldsymbol{\Psi}_{n}\right]_{m}=\left[\begin{array}{lll}
e^{-j \omega_{m} \tau_{(0,0), n}} & \cdots & e^{-j \omega_{m} \tau_{\left(N_{x}-1, N_{z}-1\right), n}}
\end{array}\right]
$$

with $m=0, \ldots, M-1$. Vector $\mathbf{r}$ can be viewed as the output of a weighted indicator function, which takes the value $\sigma_{p}$ if the $p$-th point target exists at the $(k, l)$-th pixel; otherwise, it is zero.

Eqn. (6) considers the contribution of only one antenna location. Stacking the measurement vectors corresponding to all $N$ antennas forms the vector $\mathbf{y}$,

$$
\mathbf{y}=\left[\begin{array}{llll}
\mathbf{y}_{0}^{T} & \mathbf{y}_{1}^{T} & \cdots & \mathbf{y}_{N-1}^{T}
\end{array}\right]^{T} .
$$


Eqns. (6) and (8) together yield the linear system of equations,

$$
\mathbf{y}=\Psi \mathbf{r}
$$

where

$$
\boldsymbol{\Psi}=\left[\begin{array}{llll}
\boldsymbol{\Psi}_{0}^{T} & \boldsymbol{\Psi}_{1}^{T} & \cdots & \boldsymbol{\Psi}_{N-1}^{T}
\end{array}\right]^{T}
$$

denotes the overcomplete dictionary with $M N$ rows and $N_{x} N_{z}$ columns.

Towards the objective of fast data acquisition, consider $\breve{\mathbf{y}}$, which is a vector of length $Q_{1} Q_{2}$ $(<<M N)$, consisting of elements chosen from $\mathbf{y}$ as follows,

$$
\breve{\mathbf{y}}=\boldsymbol{\Phi} \mathbf{y}
$$

where $\boldsymbol{\Phi}$ is a $Q_{1} Q_{2} \times M N$ matrix of the form,

$$
\boldsymbol{\Phi}=\operatorname{kron}\left(\boldsymbol{\vartheta}, \mathbf{I}_{Q_{1}}\right) \cdot \operatorname{diag}\left\{\boldsymbol{\varphi}^{(0)}, \ldots, \boldsymbol{\varphi}^{(N-1)}\right\}
$$

In eqn. (12), 'kron' denotes the Kronecker product, $\boldsymbol{\vartheta}$ is a $Q_{2} \times N$ measurement matrix constructed by uniformly or randomly selecting $Q_{2}$ rows of an $N \times N$ identity matrix, and $\varphi^{(n)}, n=0,1, \ldots, N-1$, is a $Q_{1} \times M$ measurement matrix constructed by uniformly or randomly selecting $Q_{1}$ rows of an $M \times M$ identity matrix. We note that $\vartheta$ determines the reduced antenna locations, whereas $\varphi^{(n)}$ determines the reduced set of frequencies corresponding to the $n$-th antenna location. We aim to solve the optimization problem,

$$
\left(P_{0}\right) \quad \min _{\mathbf{x}}\|\mathbf{x}\|_{l_{0}} \quad \text { subject to } \quad \breve{\mathbf{y}} \approx \mathbf{\Phi} \mathbf{y}
$$

where $l_{0}$ is the number of non-zero elements in $\mathbf{x}$. This problem requires an exhaustive search and, in general, its solution is not feasible. CS recovers the sparse signal $\mathbf{x}$ from $\breve{\mathbf{y}}$ by solving the following convexification of $\left(P_{0}\right)$,

$$
\left(P_{1}\right) \quad \min _{\mathbf{x}}\|\mathbf{x}\|_{l_{1}} \quad \text { subject to } \quad \breve{\mathbf{y}} \approx \mathbf{\Phi} \mathbf{y}
$$

where $\|\mathbf{x}\|_{l_{1}}=\sum_{i}\left|x_{i}\right|$. Several methods are available in the literature to solve the optimization problem $\left(P_{1}\right)$. The $l_{1}$-minimization is a convex problem and can be recast as a linear program (LP) [31]. This is the foundation for the Basis Pursuit (BP) techniques [13], [25], [32]. Alternatively, greedy methods, known as Matching Pursuit (MP), can be used to solve $\left(P_{1}\right)$ iteratively [33], [34]. In this paper, we choose Orthogonal Matching Pursuit (OMP) to solve $\left(P_{1}\right)$, which is known to provide a fast and easy to implement solution. 


\section{Sparse Representation of the Image}

The prevalent assumptions in CS-based TWRI literature are that i) the scene being imaged itself is sparse, and ii) the targets satisfy the point target model. The approach based on these assumptions, presented in Section III, will be called the conventional approach henceforth. Recently, another CS-based approach has been proposed, which employs the 2D Discrete Wavelet Transform (2D-DWT) as a sparsifying transform for through-the-wall images [30]. The introduction of the 2D-DWT overcomes the point target limitation of the conventional approach and provides the capability to deal with extended targets. Alternatively, extended targets can also be handled by the exploiting the block-sparse structure of the scene, as proposed in [35] for general CS applications. The block-sparse approach, applied to TWRI, amounts to maintaining the conventional point-target based CS model and suitably extending the recovery algorithm by assuming blocks of contiguous target pixels. In this section, we review the wavelet-based and block-sparse approaches and present the proposed sparsifying basis for interior wall and corner detection application.

\section{A. 2D Discrete Wavelet Transform}

In practical TWRI situations, medium sized targets populate more than a single pixel in the image, thus making the point target assumption no longer valid. Hence, a more appropriate basis other than just the columns of an identity matrix is desirable for dealing with extended targets. The wavelet basis is widely used for sparse representation in image compression [36], and is adequate for representing sharp variations of the image intensity. Thus, the conventional CS image formation was combined with the 2D-DWT in [30], yielding a more appropriate approach for extended target detection in CS-based TWRI.

An extensive study of the performance of different 2D-DWTs was carried out in [30], concluding that the Dual-Tree Complex Wavelet Transform (DT-CWT) is the most appropriate candidate for TWRI images. In this case, the sparsifying dictionary is given by $\mathbf{W}$, where $\mathbf{W} \in \mathbb{C}^{N_{x} N_{z} \times W}$ is the matrix spanning the vector space of the DT-CWT basis, with $W$ being the number of wavelet coefficients. The linear system of equations relating the observed data $\mathbf{y}$ and the wavelet coefficients is given by,

$$
\mathbf{y}=\Psi \mathbf{r}=\Psi \mathbf{\Psi x}
$$


where $\mathbf{x}$ is the sparse image representation expressed in the wavelet basis. The optimization of the wavelet basis for the wall detection problem is out of the scope of this paper.

\section{B. Proposed Approach: Sparsifying Dictionary for Imaging of Building Structures}

In this section, we propose a new sparsifying approach for imaging building interior structures in TWRI. The typical geometrical signatures of building interior walls and prior information about common construction practices are exploited to design two sparsifying dictionaries wellsuited to the wall and the corner detection problem which will allow us to work with far fewer measurements. First, a sparsifying dictionary based on possible wall locations is used to infer wall positions. Then, more information regarding the junctions between parallel and perpendicular walls is obtained following a similar sparsifying dictionary based on dihedral reflection response. It is noted that although the signal model considered here assumes a 2D scene, the proposed approach can be readily extended to 3D by handling reflections from wall-floor and wall-ceiling junctions in a manner similar to the wall-wall junctions. This section is divided into three parts. The first part concentrates on the proper linear representation relating the observed data with the image, whereas the second and the third part introduce the two sparsifying basis for interior structure detection applications.

1) Linear model for interior wall detection: Instead of the sensing matrix described in eqn. (10) where each antenna accumulates the contributions of all the pixels, we use an alternate sensing matrix, proposed in [37], to relate the scene vector, $\mathbf{r}$, and the observation vector, $\mathbf{y}$. This matrix underlines the specular reflections produced by the walls. Specular reflection is distinct from diffuse reflection produced by point-like targets, where incoming waves are reflected in a broad range of directions. Due to wall specular reflections, and since the array is assumed parallel to the front wall and, thus, parallel to interior walls, the rays collected at the $n$-th antenna will be produced by portions of the walls that are only in front of this antenna (see Fig. 2). The alternate matrix, therefore, only considers the contributions of the pixels that are located in front of each antenna. In so doing, the returns of the walls located parallel to the array axis are emphasized. As such, it is most suited to the specific building structure imaging problem, wherein the signal returns are mainly caused by EM reflections of exterior and interior walls. The alternate linear model can be expressed as

$$
\mathbf{y}=\hat{\Psi} \mathbf{r}
$$


where

$$
\hat{\mathbf{\Psi}}=\left[\begin{array}{llll}
\hat{\mathbf{\Psi}}_{0}^{T} & \hat{\mathbf{\Psi}}_{1}^{T} & \cdots & \hat{\mathbf{\Psi}}_{N-1}^{T}
\end{array}\right]^{T}
$$

with $\hat{\Psi}_{n}$ defined as,

$$
\left[\hat{\Psi}_{n}\right]_{m}=\left[\Im_{[(00), n]} e^{-j \omega_{m} \tau_{(0,0)}} \quad \ldots \quad \Im_{\left[\left(N_{x}-1, N_{z}-1\right), n\right]} e^{-j \omega_{m} \tau_{\left(N_{x}-1, N_{z}-1\right)}}\right]
$$

In eqn. (18), $\tau_{(k, l)}=\frac{2 \rho_{l}}{c}$ is the two-way signal propagation time associated with the downrange $\rho_{l}$ of the $(k, l)$-th pixel, and the function $\Im_{[(k, l), n]}$ works as an indicator function in the following way,

$$
\Im_{[(k, l), n]}= \begin{cases}1 & \text { if the }(k, l) \text {-th pixel is in front of the } n \text {-th antenna } \\ 0 & \text { otherwise }\end{cases}
$$

That is, if $x_{k}, x_{n}$ and $\partial x$ represent the crossrange coordinate of the $(k, l)$-th pixel, the crossrange coordinate of the $n$-th antenna location, and the crossrange sampling step, respectively, then $\Im_{[(k, l), n]}=1$ provided that $x_{k}-\frac{\partial x}{2} \leq x_{n} \leq x_{k}+\frac{\partial x}{2}$ (see Fig. 3).

2) Sparsifying basis for interior wall detection: With the synthetic array aperture parallel to the front wall, interior walls parallel to the array will dominate the image. We, therefore, focus primarily on the detection of interior walls parallel to the front wall. It is noted that the walls perpendicular to the array could return the radiated wave through multipath via involvement of the parallel walls. This issue is discussed in Section V. The number of parallel walls is typically much smaller compared to the downrange extent of the building, and thus, the decomposition of the image into horizontal walls can be considered as sparse. Note that although other indoor targets, such as furniture, humans and wall-wall corners, may be present in the scene, their projections onto the horizontal lines are expected to be negligible compared to those of the wall.

In order to obtain a linear matrix-vector relation between the scene and the horizontal projections, we define a sparsifying matrix $\mathbf{R}$ composed of possible wall locations. Specifically, each column of the dictionary $\mathbf{R}$ represents an image containing a single wall of length $l_{x}$ pixels, located at a specific crossrange and at a specific downrange in the image. Consider the crossrange to be divided into $N_{c}$ non-overlapping blocks of $l_{x}$ pixels each (see Fig. 4), and the downrange division defined by the pixel grid. The number of blocks $N_{c}$ is determined by the value of $l_{x}$, which is the minimum expected wall length in the scene. Therefore, the dimension of $\mathbf{R}$ is $N_{x} N_{z} \times N_{c} N_{z}$, where the product $N_{c} N_{z}$ denotes the number of possible wall locations. Fig. 
5 shows a simplified scheme of the sparsifying dictionary generation. The projection associated with each wall location is given by,

$$
g^{(b)}(l)=\frac{1}{l_{x}} \sum_{k \in B[b]} r(k, l)
$$

where $B[b]$ indicates the $b$-th crossrange block and $b=1, \ldots, N_{c}$. Defining

$\mathbf{g}=\left[\begin{array}{llllllllll}g^{(1)}(0) & \ldots & g^{\left(N_{c}\right)}(0) & g^{(1)}(1) & \cdots & g^{\left(N_{c}\right)}(1) & \cdots & g^{(1)}\left(N_{z}-1\right) & \cdots & g^{\left(N_{c}\right)}\left(N_{z}-1\right)\end{array}\right]^{T}$,

the linear system of equations relating the observed data $\mathbf{y}$ and the sparse vector $\mathbf{g}$ is given by,

$$
\mathbf{y}=\hat{\mathbf{\Psi}} \mathbf{R g}
$$

In practice and by the virtue of collecting signal reflections corresponding to the zero aspect angle, any interior wall outside the synthetic array extent will not be visible to the system. Finally, the reconstructed CS image in this case is obtained by first recovering the projection vector $\mathbf{g}$ using OMP and then forming the product $\mathbf{R g}$.

The projection defined in eqn. (20) clearly favors the detection of targets whose extent spreads in the crossrange image dimension. Thus, the contribution of horizontal walls will be much stronger than the contribution of point targets when using the proposed projection. This can be proven as follows. For simplicity, we assume that the crossrange pixel division matches exactly the antenna array spacing $\left(x_{k}=x_{n}\right.$ and $\partial x=\partial n$, where $\partial n$ represent the inter-element spacing of the array). Consider a single block $B[b]$ located at the $l$-th downrange, which contains a single point target with reflectivity $\sigma_{p}$. That is, out of the $l_{x}$ pixels that form $B[b]$, all are equal to zero except one which assumes the value $\sigma_{p}$. Thus, the contribution of the point target under the proposed sparsifying basis is $g_{p}^{(b)}(l)=\frac{\sigma_{p}}{l_{x}}$. On the other hand, if the block $B[b]$ contains a wall instead of a point target, all the pixels that form $B[b]$ will be equal to the wall reflectivity $\sigma_{w}$ and, thus, the contribution in this case will be $g_{w}^{(b)}(l)=\sigma_{w}$. Even considering the unusual case of $\sigma_{p}=\sigma_{w}$ (for the normal angle wall returns under consideration, $\sigma_{p}<<\sigma_{w}$ ),

$$
g_{p}^{(b)}(l)<g_{w}^{(b)}(l)
$$

It is noted that we are implicitly assuming that the extents of the walls in the scene are integer multiples of the block of $l_{x}$ pixels. In case this condition is not satisfied, the maximum error in determining the wall extent will be at the most equal to the chosen block size. In order to reduce 
this error, the chosen block size should be small. However, a smaller block size will cause the contributions from point-like targets to increase under the proposed sparsifying basis. In short, there exists a tradeoff between wall extent estimation error and the ability of the proposed basis to favor targets extended in crossrange. The follow on step of detecting reflections from wall-wall junctions can help alleviate this issue.

3) Sparsifying basis for corner detection: Once the wall locations have been determined, more information about the walls and their extent can be inferred by detecting the corners (dihedral) formed by the intersection of two walls that are perpendicular to each other. We will, therefore, search for dihedrals along the $N_{x}$ possible crossrange locations but only at the downrange, where a wall was detected in the previous stage. Thus, assuming that $N_{w}$ walls are detected, the sparsifying dictionary based on possible dihedral locations, named $\Lambda$, can be introduced with $N_{x} N_{w}$ columns, each corresponds to a corner response located at a different position. The corner response is modeled following eqns. (2) and (3).

The linear relationship between the possible dihedral locations, represented by the column vector $\nu$, and the observed data measurements is given by,

$$
\mathbf{y}=\Lambda \nu
$$

Therefore, the initial step in the proposed algorithm is to detect wall positions from the compressed data measurements using the sparse model defined in eqn. (22). Using the downranges of the detected walls, a dictionary of dihedral positions (eqn. (24)) is then used to determine the extent of the parallel walls by indicating the locations of perpendicular walls.

\section{Block-Sparsity Approach}

The sparsifying model proposed in Section IV-B takes advantage of the additional structure in the form of the non-zero entries occurring in clusters exhibited by the scene image containing interior walls. Another possible approach that can take advantage of the clustering property is the block-sparsity approach [35]. Consider the sparse representation of the TWRI data defined in eqn. (9), which operates under the point target model. Similar to Section IV-B2, in order to define block-sparsity, we view $\mathbf{r}$ as a concatenation of $N_{c} N_{z}$ blocks of length $l_{x}$, i.e. [35],

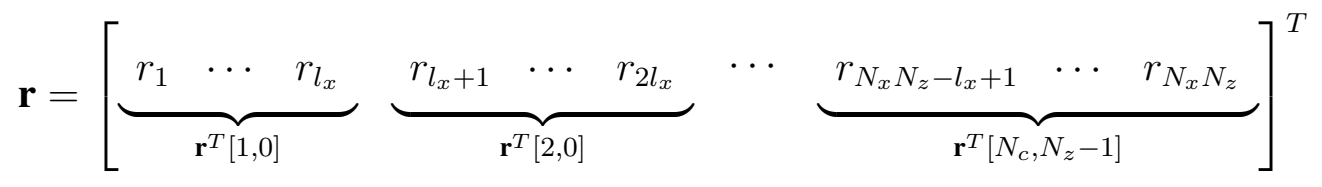


where $\mathbf{r}^{T}[b, l]$ denotes the $b$-th crossrange block located at the $l$-th downrange. For building layout detection, image $\mathbf{r}$ is block $K$-sparse, i.e., it has non-zero Euclidean norm for at most $K$ blocks. Similarly, we can represent $\Psi$ as a concatenation of column-blocks $\Psi[b, l]$ of size $M N \times l_{x}$,

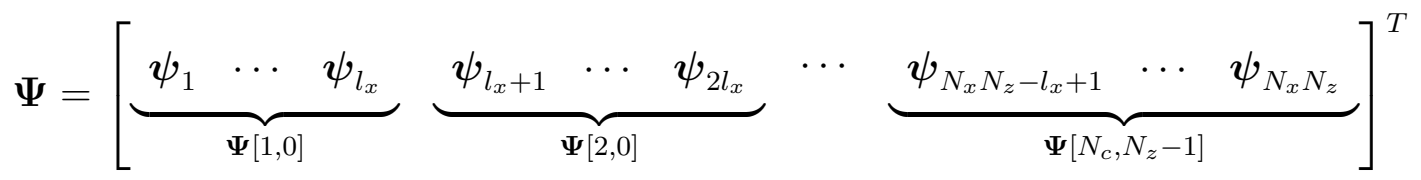

Once the formulation is expressed in a block form, the block extension of OMP (BOMP) can be applied to recover the sparse image $\mathbf{r}$ [35].

\section{Multipath EFFects}

EM propagation in highly scattering environment suffers from multipath effects. The multipath signals can cause ghost targets, which increase the number of false alarms reported by the system. The problem of multipath propagation in TWRI has been investigated in recent years in [38][40]. The image can be improved by correcting the ghosting problem while retaining the true targets, provided a good EM model of the propagation environment is available, which, in turn, requires knowledge of the building layout.

For the building layout detection problem considered in this paper and excluding the corner junction caused by two walls, two types of multipath returns or reverberations are prevalent, namely, building reverberation and front wall reverberation. Building reverberation is a result of multiple reflections of the transmitted signal off interior walls within the building, whereas multiple reflections within the front wall constitute the front wall reverberation. In this section, we investigate the effects of the reverberations on the building layout detection problem. The walls are assumed to be smooth with specular reflection. Fig. 6 shows the trajectory of one possible twice-bounced component of the building reverberation. Due to the specular nature of wall reflections, which requires the angle of incidence to be equal to the angle of reflection, it is clear from Fig. 6 that a twice-bounced reverberation component will not return to the transceiver location, except in the case of a double-bounced reflection from the corners. The corner reflections have been separately incorporated into the signal model in Section II and are thus not considered in this Section. There exist higher order multipath components with multiple bounces from other walls that may arrive at the transceiver location, but these paths are 
weaker due to the secondary reflections and the higher path loss. As such, their contribution is disregarded in this work. Based on the aforementioned observations, building reverberation will not pose a problem for the proposed method for interior layout detection.

On the other hand, front wall reverberation, depicted in Fig. 7-path(b), would cause replicas of the front wall to appear in the image at downranges behind the wall. We note that this issue will be less pronounced in wall materials, such as concrete, in which the radar signal undergoing multiple bounces within the wall suffers a higher degree of attenuation. We demonstrate this through simulation examples in Section VI.

\section{Simulation and Experimental Results}

\section{A. Simulation Results}

In this section, we evaluate the performance of the proposed scheme using synthesized data. A stepped-frequency signal consisting of 335 frequencies covering the 1 to $2 \mathrm{GHz}$ frequency band was used for interrogating the scene. A monostatic synthetic aperture array, consisting of 71element locations with an inter-element spacing of $2.2 \mathrm{~cm}$, was employed. The array was located parallel to a $1.6 \mathrm{~m}$-wide front wall, and centered at $0 \mathrm{~m}$ in crossrange at a standoff distance of $2.42 \mathrm{~m}$. The scene behind the front wall contained two interior walls, a back wall, and a single point target. The first interior wall extended from $-0.78 \mathrm{~m}$ to $-0.29 \mathrm{~m}$ in crossrange at a downrange of $3.37 \mathrm{~m}$, while the second wall was located at $5.12 \mathrm{~m}$ downrange, extending from $0.29 \mathrm{~m}$ to $0.78 \mathrm{~m}$ in crossrange. The $1.6 \mathrm{~m}$ wide back wall was located at $6.24 \mathrm{~m}$ and was aligned with the front wall in crossrange. To simulate different wall materials, we considered different reflectivities for interior $(\sigma=0.85)$ and exterior walls $(\sigma=1)$. The two interior walls and the back wall have corner reflectors on their extremities to emulate the junctions between parallel and perpendicular walls. The point target was located at $(0.02,4.24) \mathrm{m}$. Fig. 8 depicts the geometry of the simulated scene. The region to be imaged is chosen to be $5.65 \mathrm{~m}$ (crossrange) $\times 4.45 \mathrm{~m}$ (downrange), centered at $(0,4.23) \mathrm{m}$, and is divided into $128 \times 128$ pixels.

Fig. 9 shows the through-the-wall radar image obtained with the backprojection algorithm, described in eqn. (5), using all $M N$ observations. In this figure and all subsequent figures in this section, we plot the image intensity with the maximum intensity value in each image normalized to $0 \mathrm{~dB}$. The four walls and the point target are clearly visible in Fig. 9. For sparsity-based imaging, we consider only $6.4 \%$ of the full data volume ( $25 \%$ uniformly selected frequencies and 
$25 \%$ uniformly chosen sensor locations). Fig. 10 depicts the CS image reconstruction obtained with the following three approaches: conventional CS approach, wavelet-based CS approach and the proposed approach for wall detection. The number of wavelets coefficients, $W$, was set to 65536 , which corresponds to a DT-CWT with depth of decomposition equal to 4 . For the proposed approach, the number of blocks, $N_{c}$, was set equal to 3 and the size of each block was chosen to be 1 pixel in downrange and $l_{x}=12$ pixels in crossrange, which corresponds to a minimum wall length of $0.53 \mathrm{~m}$. In this simulation, the walls present in the scene lie along the pre-defined block grid. Later, we will show a case where this assumption is not satisfied. We forced the number of iterations of the OMP to be equal to 8 in all cases, which means that the output of the OMP will be a sparse vector of 8 non-zero components. Fig. 10(a) shows the image reconstructed with the conventional CS-based imaging with no sparsifying basis. Since the walls are extended targets and appear dense under the point target model, all the degrees of freedom are used up in reconstructing the front wall. Thus, the conventional approach fails to recover the building structure. The corresponding reconstructed scene obtained with the wavelet approach is shown in Fig. 10(b). Although this approach is more suitable for representing extended targets than the point target model, it is clearly not enough to reconstruct the interior building structure with 8 non-zero wavelet coefficients. Finally, Fig. 10(c) and Fig. 10(d), respectively, show the resulting image of the proposed wall detection stage and the proposed corner detection stage. It is evident that the image produced by the proposed wall detector, which is shown in Fig. 10(c), has adequately reconstructed the structure of the building. Wall corner detections, as shown in Fig. $10(\mathrm{~d})$, refine the detection of building structures by indicating the extents of the walls detected in Fig. 10(c).

Considering the scene itself to be sparse and making use of the block-sparsity in the reconstruction algorithm with BOMP yields the image shown in Fig. 11. We observe that not only some portions of the back and front wall are missing from the image, but also a few false blocks have been reconstructed. Basically, BOMP reconstructs the most prominent blocks that appear in Fig. 9, which includes the artifacts resulting from range sidelobes of the front wall response. These deficiencies can be attributed to the underlying point target model.

In general, the wall extension may not fit the block grid defined by the sparsifying basis for interior wall detection. In the following example, we consider different extents for the two interior walls of the previous example. In particular, the first interior wall extends now from 
$-0.78 \mathrm{~m}$ to $-0.56 \mathrm{~m}$ in crossrange at the same downrange, while the second wall is located as before at $5.12 \mathrm{~m}$ downrange, but extending from $0.02 \mathrm{~m}$ to $0.78 \mathrm{~m}$ in crossrange. Fig. 12 depicts the layout of the simulated scene. According to these wall measurements, the first interior wall occupies half a block, whereas the second wall occupies one and a half block. Again, working with the same $6.4 \%$ of the measurements, Fig. 13(a) and Fig. 13(b) show the recovered CS images from the wall detection step and the corner detection step, respectively. Fig. 13(a) shows how the wall that is occupying one and a half block is now recovered as a two block wall, and the half block wall is recovered as a complete block wall. These initial wall estimates can be refined and corrected with the corner detection, which accurately determines the extent of each wall.

In order to provide a quantitative analysis of the performance of the proposed approach, we use the Earth Mover's Distance (EMD) [41], [42]. This is a popular metric for images and is used in image similarity. Limiting the error in sparsely recovered images to $l_{p}$ norms is quite inconvenient because they do not accurately represent perceptual differences between images [43], [44]. Intuitively, given two images, the EMD reflects the minimal amount of work that must be performed to transform one image into the other. Thus, the EMD naturally extends the notion of a distance between single elements to that of distance between sets or distributions [45]. The EMD metric was used in a CS framework in [43], [44]. Plots showing the EMD of the proposed wall detection approach and the proposed corner detection approach versus signalto-noise ratio (SNR) are provided in Fig. 14 and Fig. 15, respectively. In order to generate these plots, white Gaussian noise was added to the simulated data measurements. Both Fig. 14 and Fig. 15 make evident the degradation of the proposed techniques in terms of detection capability due to the reduction in the data volume. Fig. 14(a) and Fig. 15(a) correspond to reduction of the frequency observations, while the number of sensors is reduced in Fig. 14(b) and Fig. 15(b). In both cases, the EMD increases as the SNR decreases. Furthermore, for a given SNR, the EMD increases with decreasing data volume.

Finally, we investigate the effect of the echoes produced by front wall reverberation. In the simulation, we consider the first-order reverberation of the front wall plotted in Fig. 7path(b). Higher-order reverberation are, in general, weaker compared to the first-order one due to secondary reflections and high attenuation in the wall material. Therefore, these paths are not taken into account in our simulation. The simulated scene is the same described at the beginning 
of this section. We use $6.4 \%$ of the data and the number of iterations is set to 8 for the wall detection step, as before. Fig. 16 shows the performance of the proposed approach for two types of front wall. Fig. 16(a) considers a $0.15 \mathrm{~m}$ thick solid concrete wall with dielectric constant of 7.66, whose first-order reverberation attenuation is set equal to $30 \mathrm{~dB}$. With this type of wall, the reverberation of the front wall is weak and thus, the proposed approach does not produce replicated images of the front wall. On the other hand, Fig. 16(b) considers a less attenuative wall (a $0.15 \mathrm{~m}$ thick adobe brick front wall with a dielectric constant of 3.2), whose reverberation attenuation is equal to $15 \mathrm{~dB}$. In this case, the algorithm reconstructs the replica of the front wall and misses the back wall because the reverberation causes a ghost whose contribution is higher than the back wall of the room.

\section{B. Experimental Results}

A through-the-wall SAR system was set up in the Radar Imaging Lab, Villanova University. The system and signal parameters are the same as those for the simulated data. The scene consisted of two parallel plywood walls, each $2.25 \mathrm{~cm}$ thick, $1.83 \mathrm{~m}$ wide, and $2.43 \mathrm{~m}$ high. Both walls were centered at $0 \mathrm{~m}$ in crossrange. The first and the second walls were located at respective distances of $3.25 \mathrm{~m}$ and $5.1 \mathrm{~m}$ from the antenna baseline. Fig. 17 depicts the geometry of the experimental scene. The region to be imaged is chosen to be the same as in Section VI-A.

Fig. 18 shows the conventional backprojection imaging result using the full dataset, wherein the two walls can be clearly seen. For the CS approach, instead of measuring all 335 frequencies at all 71 antenna locations, we use a uniform subset of only 84 frequencies at each of the 18 uniformly spaced antenna locations, which represents $6.4 \%$ of the full data volume. The CS reconstructed image is shown in Fig. 19. We note in Fig. 19 that the proposed algorithm for wall detection was able to reconstruct both walls. However, it can be observed in Fig. 18 that ghosts walls appear immediately behind each true wall position. These ghosts are attributed to the dihedral-type reflections from the wall-floor junctions.

Fig. 20 depicts the geometry of another experimental scene which considers the same two walls as before, together with a perpendicular wall which is located midpoint along the extent and connected to the two parallel walls. The perpendicular wall is also made of plywood and is $3.8 \mathrm{~cm}$ thick. Fig. 21 shows the conventional backprojection imaging result using the full dataset. We observe from this figure that the vertical wall is casting a shadow on part of the back wall. 
Using the same reduced set of measurements of 84 frequencies at 18 different antenna locations, the wall detection approach proposed in this paper produces the CS reconstructed image shown in Fig. 22(a). Clearly, the reconstruction misses the part of the back wall that is obstructed by the new perpendicular wall. Two corner features are created by the junction of the back wall and the perpendicular wall. However, Fig. 22(b) shows that the proposed corner detection approach fails in correctly detecting these two corners. This is due to the fact that the synthetic array is centered at the crossrange location of the perpendicular wall and its extent and standoff distance are such that most of the antennas pick up a weak response from the corners. This example confirms that the two steps of wall and corner detection must work in concert and corner detection by itself may not be sufficient for building layout determination.

\section{CONCLUSION}

A sparsity-based approach for imaging of interior building structure was presented. The proposed technique makes use of the prior information about building construction practices of interior walls to both devise an appropriate linear model and design a sparsifying dictionary based on the expected wall alignment relative to the radar's scan direction. In a follow on step for determining the extents of the previously detected parallel walls and for locating perpendicular walls, a sparsifying dictionary based on the possible corner reflector locations is used to detect wall-wall junctions. The proposed approach provides reliable determination of building layouts, while achieving substantial reduction in data volume. Matlab simulation based results depicted the improved performance of the proposed method when compared to backprojection, conventional point-target based CS, and CS employing wavelet sparsifying basis. Moreover, the proposed approach was shown to overcome the limitations of the recently proposed block-sparsity model when applied to building interior layout detection. Results based on laboratory experiments were also provided. In particular, a structure composed of two parallel and one perpendicular walls was considered to bring about the corner reflections. The results showed that the two steps of wall and corner detections must work in concert for reliable determination of the building interior structure.

\section{REFERENCES}

[1] M. G. Amin (Ed.), "Special issue on Advances in Indoor Radar Imaging," J. Franklin Inst., vol. 345, no. 6, pp. 556-722, Sep, 2008. 
[2] M. G. Amin (Ed.), “Through-the-Wall Radar Imaging,” CRC Press, Boca Raton, FL, 2010.

[3] M. G. Amin and K. Sarabandi (Eds.), "Special issue on Remote Sensing of Building Interior," IEEE Trans. Geosci. Remote Sens., vol. 47, no. 5, pp. 1270-1420, May, 2009.

[4] C. P. Lai and R. M. Narayanan, "Through-wall imaging and characterization of human activity using ultrawideband (UWB) random noise radar," Proc. SPIE Symp. Defense and Security, Sensors, and Command, Control, Communications, and Intelligence (C3I) Technologies IV Conference, vol. 5778, pp. 186-195, 2005.

[5] F. Soldovieri, G. Prisco, and R. Solimene, “A multi-array tomographic approach for Through-Wall Imaging," IEEE Trans. Geosci. Remote Sens., vol. 46, no. 4, pp. 1192-1199, 2008.

[6] L. P. Song, C. Yu, and Q. H. Liu, “Through-wall imaging (TWI) by radar: 2-D tomographic results and analyses," IEEE Trans. Geosci. Remote Sens., vol. 43, no. 12, pp. 2793-2798, 2005.

[7] F. Ahmad and M. G. Amin, "A noncoherent radar system approach for through-the-wall imaging," Proc. SPIE Symp. Defense and Security, Sensors, and Command, Control, Communications, and Intelligence (C3I) Technologies IV Conference, vol. 5778, pp. 196-207, 2005.

[8] F. Ahmad, M. G. Amin, and S. A. Kassam, "A Beamforming Approach to Stepped-Frequency Synthetic Aperture Throughthe-Wall Radar Imaging," IEEE Int. Workshop on Computational Advances in Multi-Sensor Adaptive Processing, vol. 345, pp. 24-27, 2005.

[9] F. Ahmad, M. G. Amin, and P. D. Zemany, "Dual-Frequency Radars for Target Localization in Urban Sensing," IEEE Trans. Aerosp. Electronic Syst., vol. 45, no. 4, pp. 1598-1609, Oct, 2009.

[10] H. Burchett, “Advances in Through Wall Radar for Search, Rescue and Security Applications," Inst. of Eng. and Tech. Conf. Crime and Security, London, UK, pp. 511-525, Jun, 2006.

[11] D.L. Donoho, “Compressed sensing,” IEEE Trans. Inf. Theory, vol. 52, no. 4, pp. 1289-1306, Apr, 2006.

[12] E. J. Candes and M. B. Wakin, "An Introduction to Compressed Sampling," IEEE Signal Process. Mag., vol. 25, no. 2, pp. 21-30, March, 2008.

[13] E. J. Candes and T. Tao, "Near Optimal Signal Recovery from Random Projections: Universal Encoding Strategies," IEEE Trans. Inf. Theory, vol. 52, no. 12, pp. 5406-5425, Dec, 2006.

[14] F. Ahmad (Ed.), Compressive Sensing, Proc. of SPIE, vol. 8365, SPIE, Bellingham, WA, 2012.

[15] L. He, S.A. Kassam, F. Ahmad, and M.G. Amin, "Sparse stepped-frequency waveform design for through-wall radar imaging," Int. Waveform Diversity and Design Conf., Lihue, HI, Jan, 2006.

[16] E.M. Lavely, Y. Zhang, E.H. Hill III, Y-S. Lai, P. Weichman, and A. Chapman, "Theoretical and experimental study of through-wall microwave tomography inverse problems," J. Franklin Inst., vol. 345, no. 6, pp. 592-617, 2008.

[17] M.M. Nikolic, M. Ortner, A. Nehorai, and A.R. Djordjevic, “An Approach to Estimating Building Layouts Using Radar and Jump-Diffusion Algorithm,” IEEE Trans. Antennas and Propag., vol. 57, no. 3, pp. 768-776, Mar, 2009.

[18] G. Hickman and J.L. Krolik, "A Graph-Theoretic Approach to Constrained Floor Plan Estimation From Radar Measurements," IEEE Trans. Signal Process., vol. 57, no. 5, pp. 1877-1888, May, 2009.

[19] C. Le, T. Dogaru, L. Nguyen, and M.A. Ressler, "Ultrawideband (uwb) radar imaging of building interior: Measurements and predictions," IEEE Trans. Geosci. Remote Sens., vol. 47, no. 5, pp. 1409-1420, May, 2009.

[20] N. Subotic, E. Keydel, J. Burns, A. Morgan, K. Cooper, B. Thelen, B. Wilson, W. Williams, S. McCarty, B. Lampe, B. Mosher, and D. Setterdahl, "Parametric reconstruction of internal building structures via canonical scattering mechanisms," IEEE Int. Conf. Acoustics, Speech and Signal Processing (ICASSP), Las Vegas, USA, 2008. 
[21] E. Ertin and R.L. Moses, "Through-the-Wall SAR Attributed Scattering Center Feature Estimation," IEEE Trans. Geosci. Remote Sens., vol. 47, no. 5, pp. 1338-1348, May, 2009.

[22] A. C. Gurbuz, J.H. McClellan, and W.R. Scott, "Compressive Sensing of underground structures using GPR," Digital Signal Processing, vol. 22, no. 1, pp. 66-73, Jan, 2012.

[23] M. Aftanas and M. Drutarovsky, "Imaging of the Building Contours with Through the Wall UWB Radar System," Radioengineering Journal, vol. 18, no. 3, pp. 258-264, 2009.

[24] M. G. Amin and F. Ahmad, "Wideband Synthetic Aperture Beamforming for Through-the-Wall Imaging," IEEE Signal Process. Mag., vol. 25, no. 4, pp. 110-113, Jul, 2008.

[25] E. J. Candes, J. Romberg, and T. Tao, "Robust Uncertainty Principles: Exact Signal Reconstruction From Highly Incomplete Frequency Information,” IEEE Trans. Inf. Theory, vol. 52, no. 2, pp. 489-509, 2006.

[26] Y.S. Yoon and M. G. Amin, “Compressed Sensing Technique for High-Resolution Radar Imaging," Proc. SPIE, vol. 6968, pp. 69681A-1-69681A-10, 2008.

[27] Q. Huang, L. Qu, B. Wu, and G. Fang, "UWB Through-Wall Imaging based on Compressive Sensing," IEEE Trans. Geosci. Remote Sens., vol. 48, no. 3, pp. 1408-1415, 2010.

[28] Y.S. Yoon and M. G. Amin, “Through-the-Wall Radar Imaging Using Compressive Sensing Along Temporal Frequency Domain," IEEE Int. Conf. Acoustics, Speech and Signal Processing (ICASSP), Dallas, USA, Mar, 2010.

[29] M.G. Amin, F. Ahmad, and W. Zhang, "Target RCS exploitations in compressive sensing for through wall imaging," 5th Int. Waveform Diversity and Design Conf., Niagara Falls, Canada, Aug., 2010.

[30] M. Leigsnering, C. Debes, and A.M. Zoubir, "Compressive Sensing in Through-the-Wall Radar Imaging," IEEE Int. Conf. Acoustics, Speech and Signal Processing (ICASSP), Prague, Czech Republic, pp. 4008-4011, 2011.

[31] S. Boyd and L. Vandenberghe, "Convex Optimization," Cambridge University Press, 2004.

[32] S. S. Chen, D. L. Donoho, and M. A. Saunders, "Atomic Decomposition by Basis Pursuit," SIAM J. Scientific Computing, vol. 20, no. 1, pp. 33-61, 1999.

[33] S. Mallat and Z. Zhang, "Matching Pursuit with Time-Frequency Dictionaries," IEEE Trans. Signal Process., vol. 41, no. 12, pp. 3397-3415, 1993.

[34] J. A. Tropp, “Greed is Good: Algorithmic Results for Sparse Approximation," IEEE Trans. on Inf. Theory, vol. 50, no. 10, pp. 2231-2242, Oct, 2004.

[35] Y.C. Eldar, P. Kuppinger, and H. Bolcskei, "Block-Sparse Signals: Uncertainty Relations and Efficient Recovery," IEEE Trans. Signal Process., vol. 58, no. 6, pp. 3042-3054, Jun, 2010.

[36] S. Mallat, “A Wavelet Tour of Signal Processing," Academic Press, 3rd edition, Dec, 2008.

[37] F. Ahmad and M. G. Amin, "Partially Sparse Reconstruction of Behind-the-Wall Scenes," Proc. SPIE Symp. Defense, Security, and Sensing, Compressive Sensing Conference, Baltimore, MD, vol. 8365, Apr, 2012.

[38] P. Setlur, M. Amin, and F. Ahmad, "Multipath Model and Exploitation in Through-the-Wall and Urban Radar Sensing," IEEE Trans. Geosci. Remote Sens., vol. 49, no. 10, pp. 4021-4034, Oct, 2011.

[39] T. Dogaru and C. Le, "SAR Images of Rooms and Buildings based on FDTD Computer Models," IEEE Trans. Geosci. Remote Sens., vol. 47, no. 5, pp. 1388-1401, May, 2009.

[40] R.J. Burkholder, "Electromagnetic Models for Exploiting Multi-Path Propagation in Through-Wall Radar Imaging," Int. Conf. Electromagnetics in Advanced Applications (ICEAA), Torino, Italy, Sep, 2009.

[41] Y. Rubner, C. Tomasi, and L.J. Guibas, “A Metric for Distributions with Applications to Image Databases," IEEE Int. Conf. Computer Vision, Bombay, India, Jan, 1998. 
[42] Y. Rubner, "Perceptual Metrics for Image Database Navigation," Ph.D. thesis, Stanford University, USA, May, 1999.

[43] R. Gupta, P. Indyk, and E. Price, “Sparse Recovery for Earth Mover Distance," Conf. Communication, Control, and Computing (Allerton), Sep, 2010.

[44] K. Krueger, J.H. McClellan, and W.R. Scott, Jr., "3-D imaging for groung penetrating radar using compressive sensing with block-toeplitz structures," IEEE 7th Sensor Array and Multichannel Signal Process. Workshop, Hoboken, NJ, Jun, 2012.

[45] Y. Rubner, C. Tomasi, and L.J. Guibas, "The Earth Mover's Distance as a Metric for Image Retrieval," Int. Journal Computer Vision, vol. 40, no. 2, pp. 99-121, 2000. 


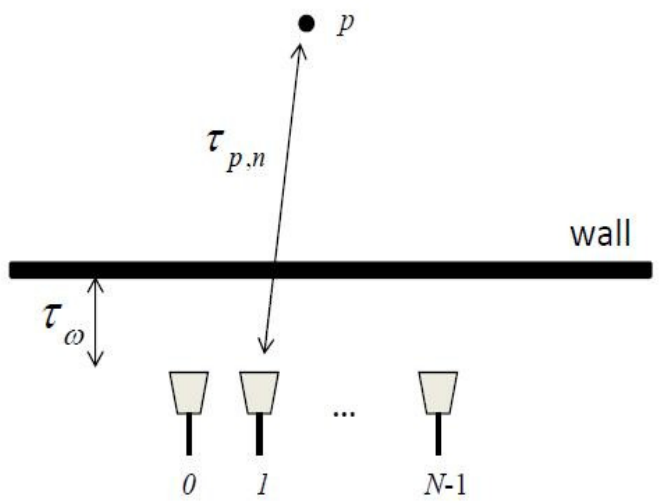

Fig. 1. Data collection using a synthetic array in through-the-wall radar imaging.

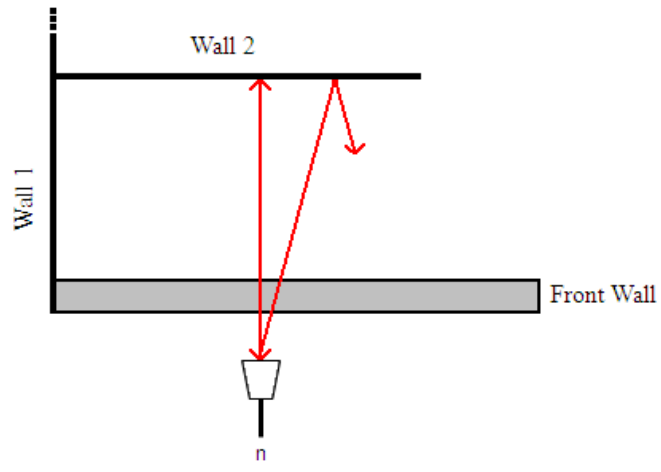

(a)

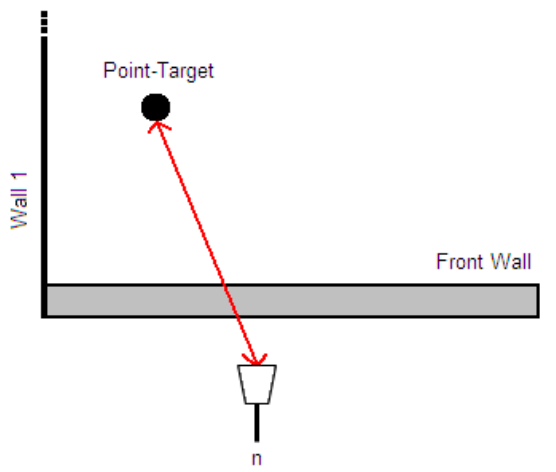

(b)

Fig. 2. Types of reflection in TWRI: (a) Specular reflection produced by walls, (b) Diffuse reflection produced by point-like targets. 


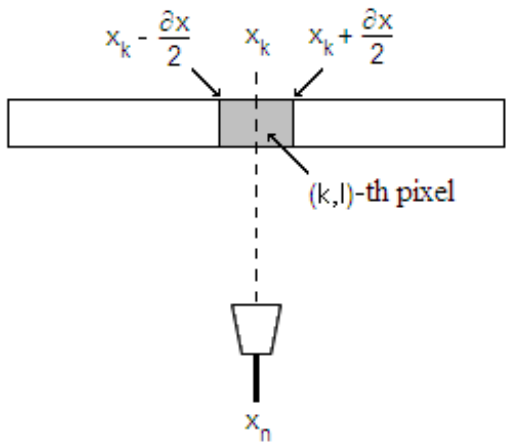

Fig. 3. Indicator function $\Im_{[(k, l), n]}$ : It takes value equal to 1 provided that $x_{k}-\frac{\partial x}{2} \leq x_{n} \leq x_{k}+\frac{\partial x}{2}$.

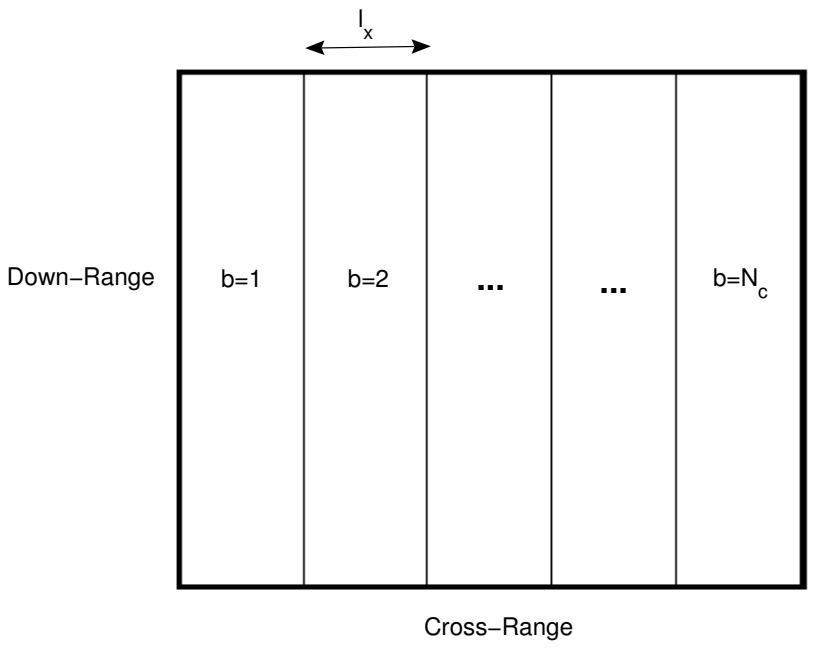

Fig. 4. Crossrange division: the image is divided into $N_{c}$ crossrange blocks of $l_{x}$ pixels. 


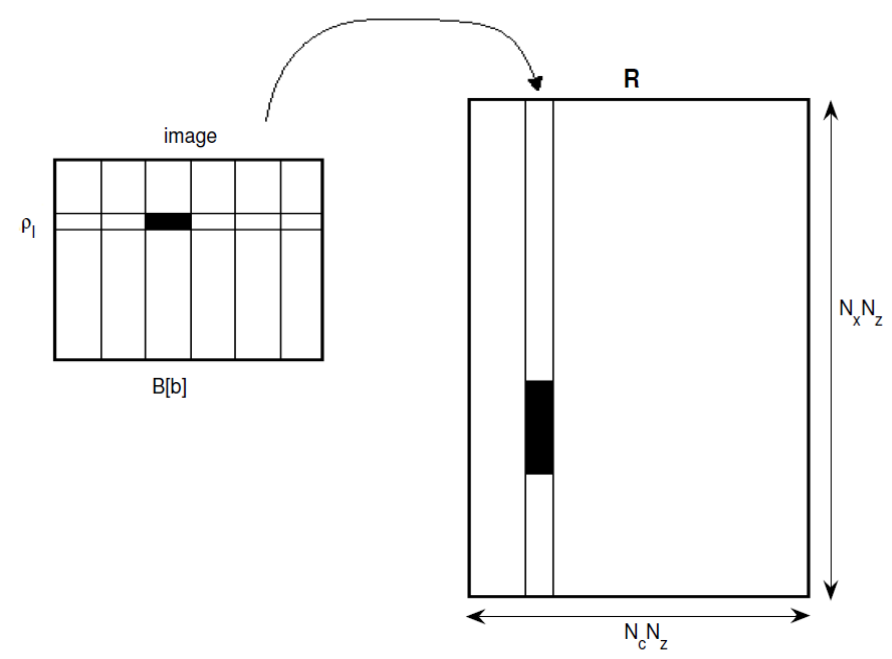

Fig. 5. Sparsifying dictionary generation.

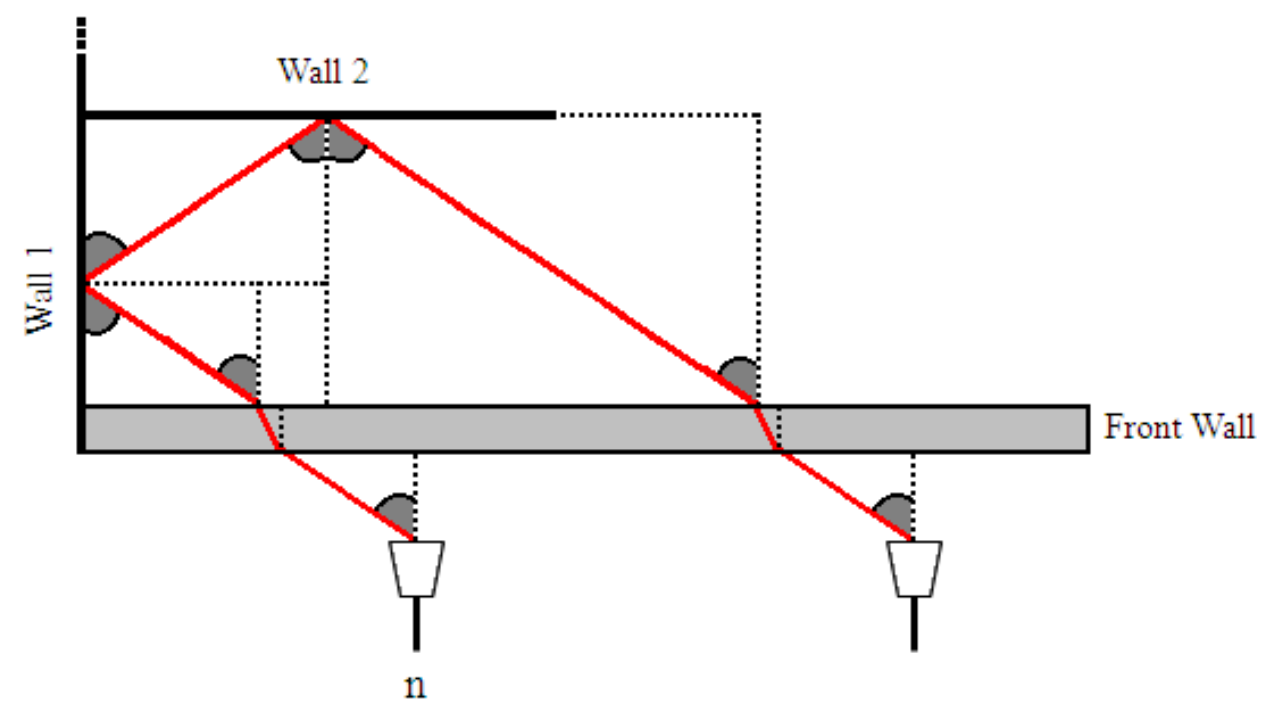

Fig. 6. Geometry depicting Building Reverberation (two bounces). 


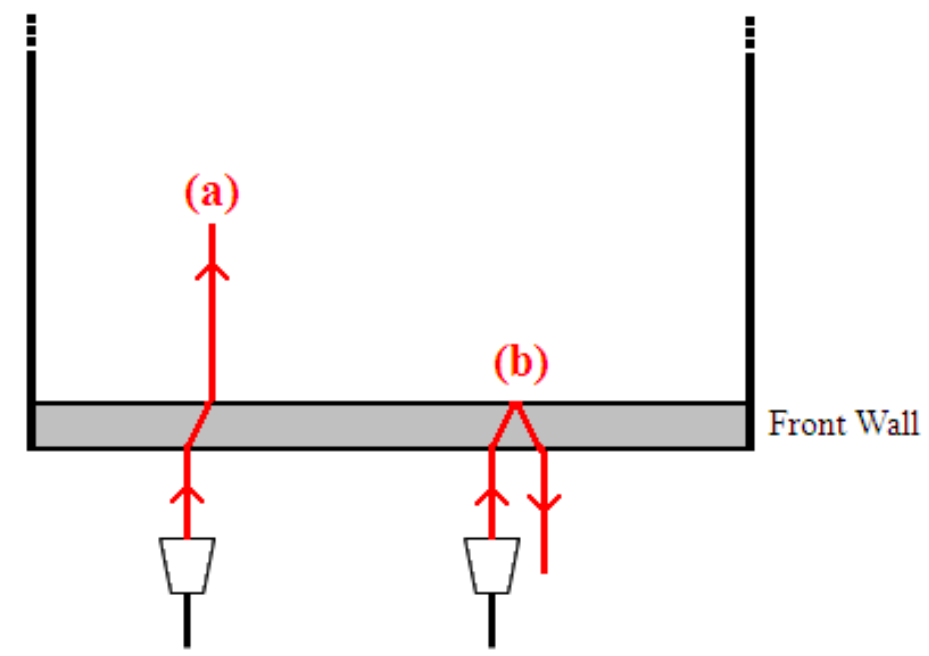

Fig. 7. Geometry depicting wall reverberation: propagation through wall described by path (a) and first-order reverberation described by path (b).

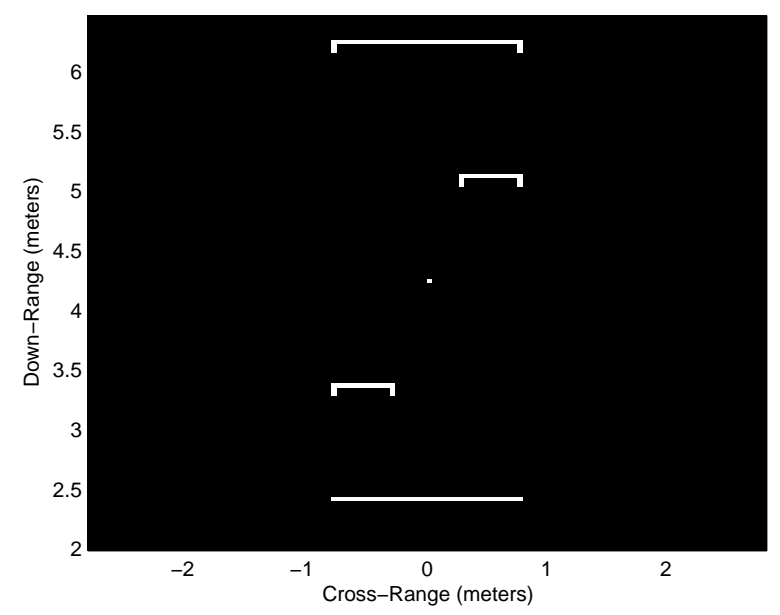

Fig. 8. Geometry of the simulated scene 


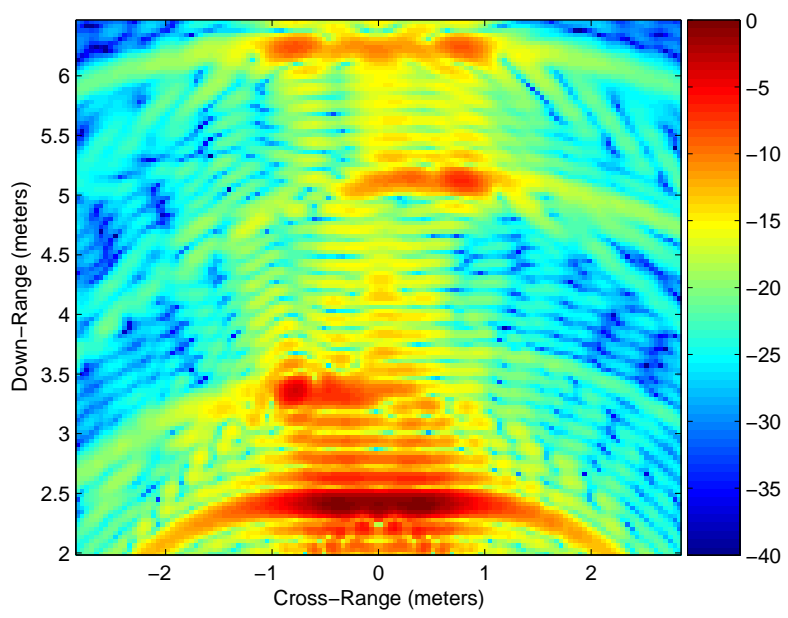

Fig. 9. Through-the-wall radar image using conventional backprojection with $100 \%$ data. 


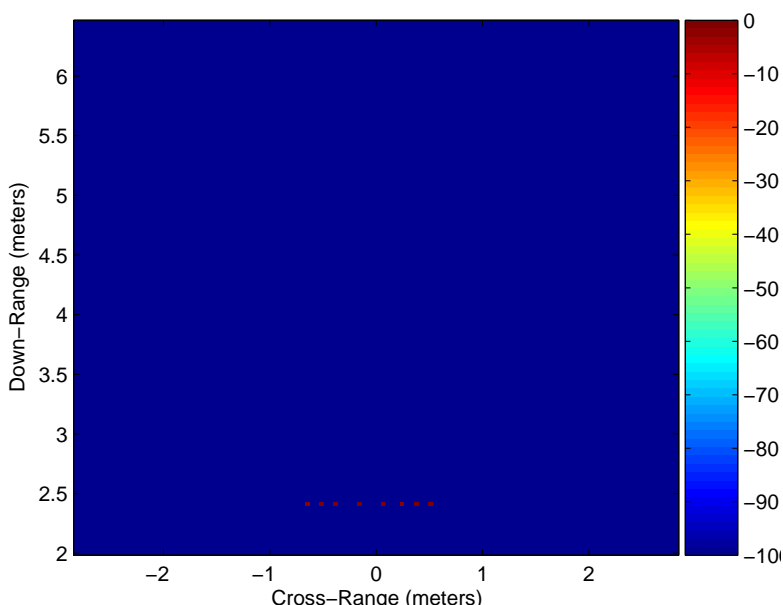

(a)

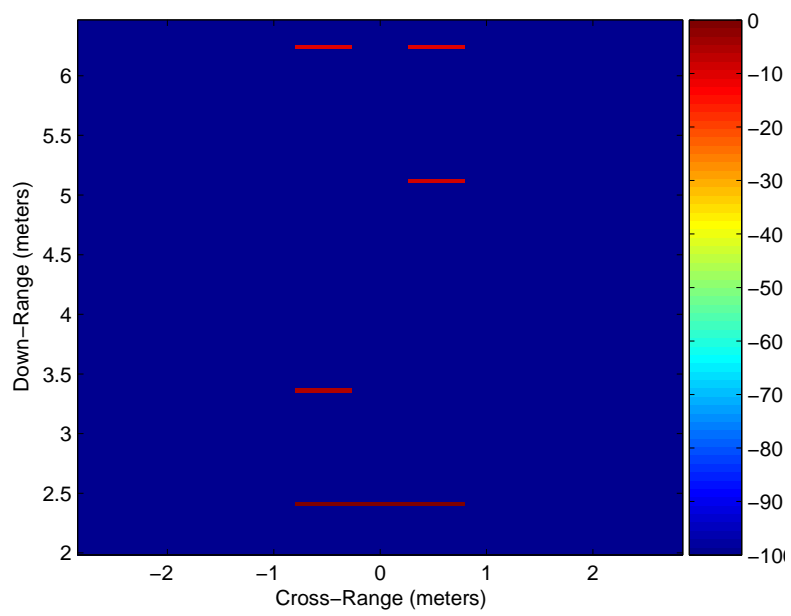

(c)

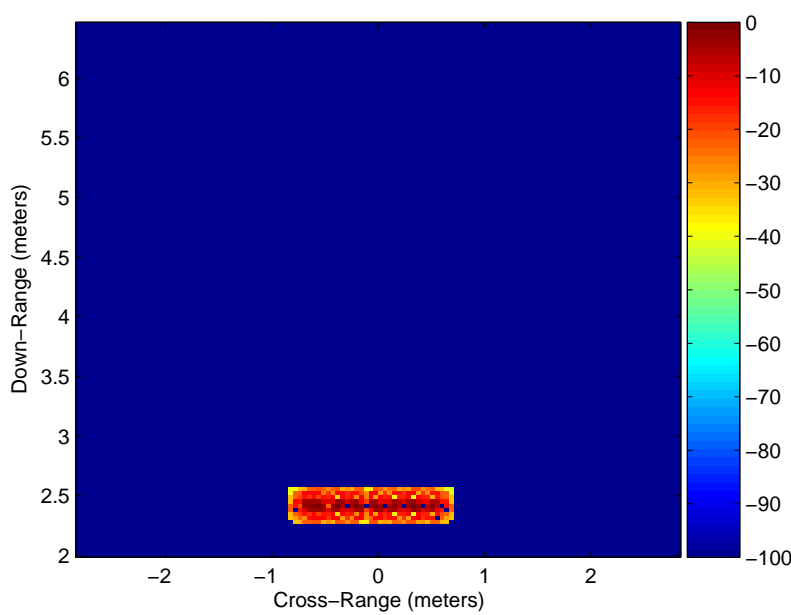

(b)

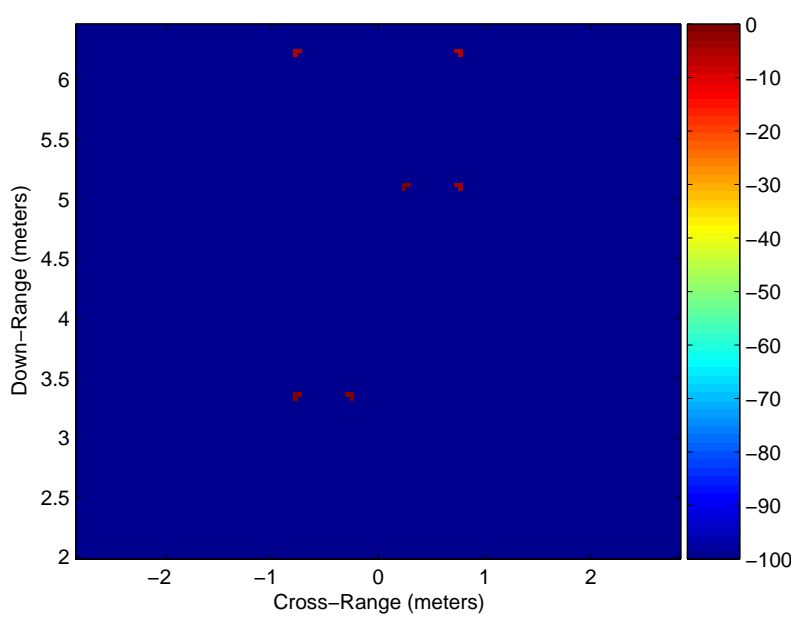

(d)

Fig. 10. Reconstructed image from the recovered sparse vector using OMP and 6.4\% data: (a) Conventional CS, (b) 2D-DWT based CS, (c) Proposed apprach for wall detection, (d) Proposed approach for corner detection. 


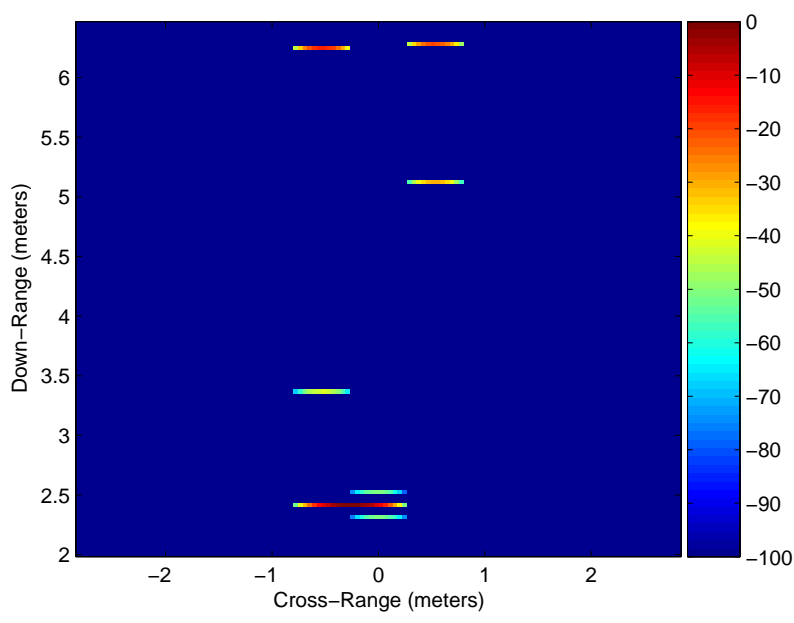

Fig. 11. Reconstructed image using BOMP and $6.4 \%$ data. The model used is $\breve{\mathbf{y}}=\boldsymbol{\Phi} \boldsymbol{\Psi} \mathbf{r}$.

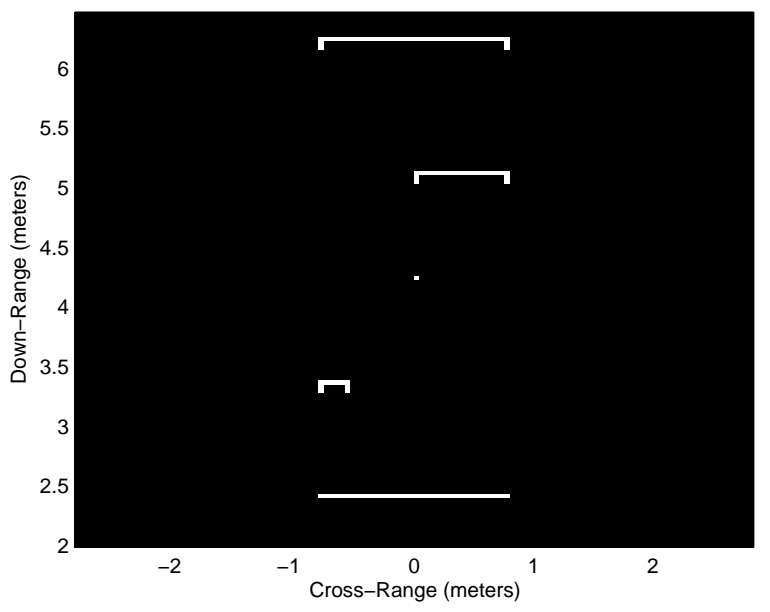

Fig. 12. Geometry of the simulated scene 


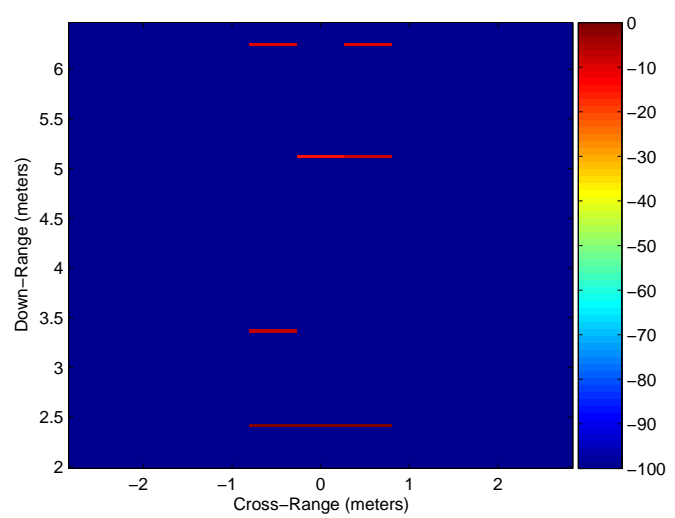

(a)

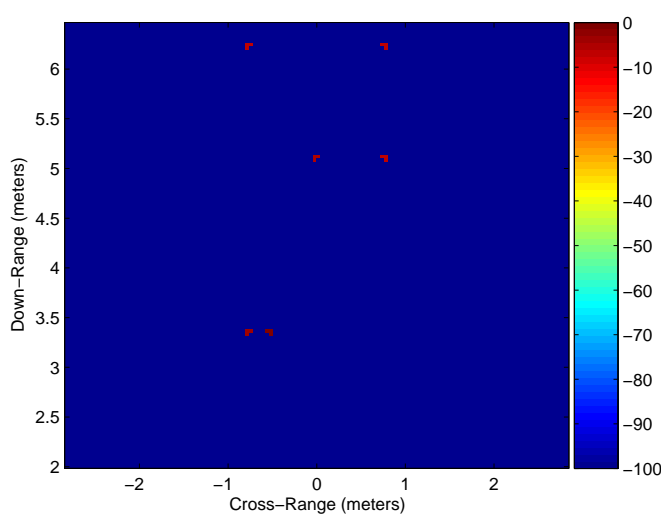

(b)

Fig. 13. Reconstructed image from the recovered sparse vector using OMP and $6.4 \%$ data: (a) Proposed approach for wall detection, (b) Proposed approach for corner detection.

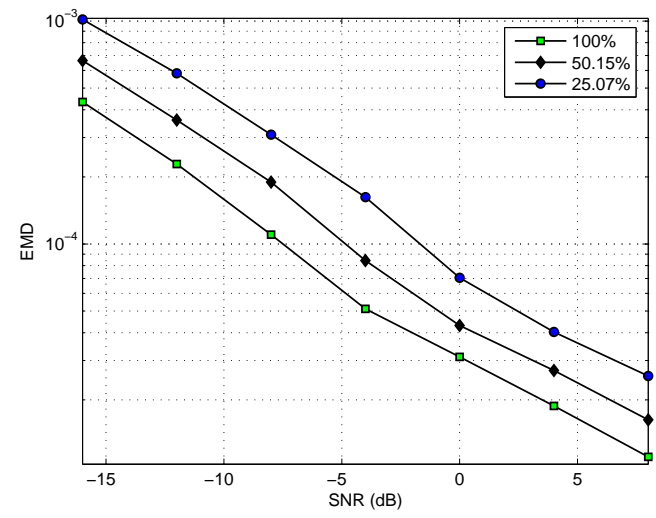

(a)

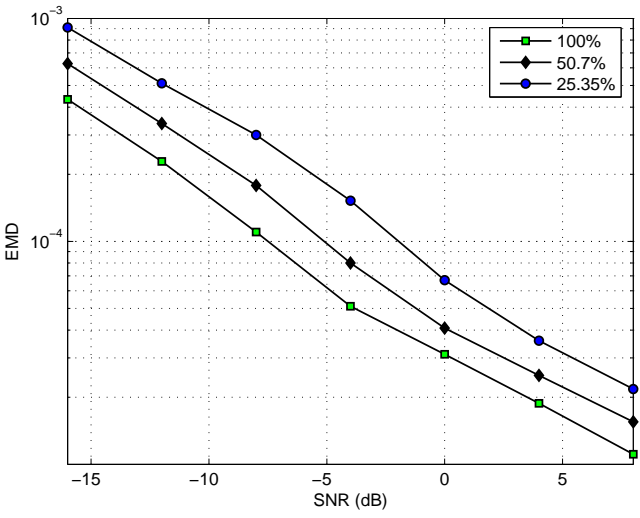

(b)

Fig. 14. EMD vs. SNR for the wall detection approach: (a) frequency compression, (b) antenna compression. 


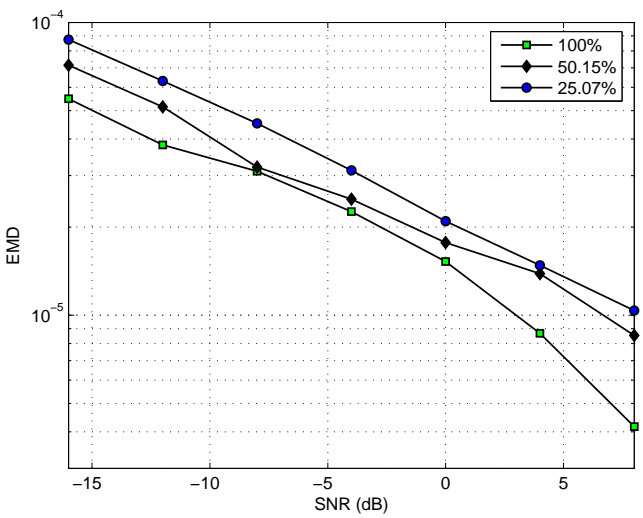

(a)

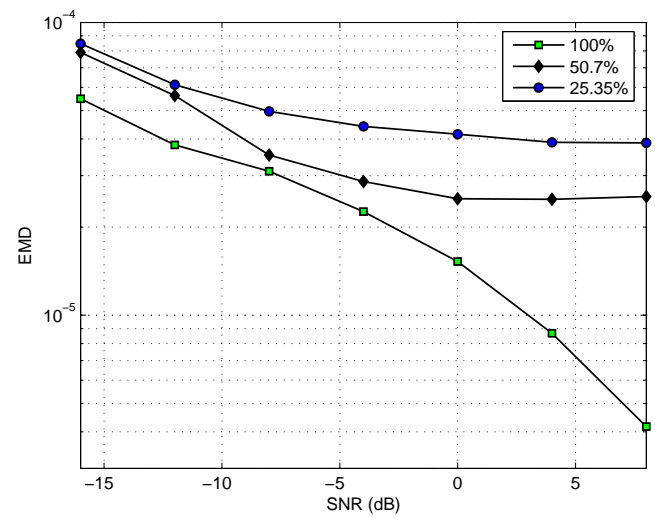

(b)

Fig. 15. EMD vs. SNR for the corner detection approach: (a) frequency compression, (b) antenna compression.

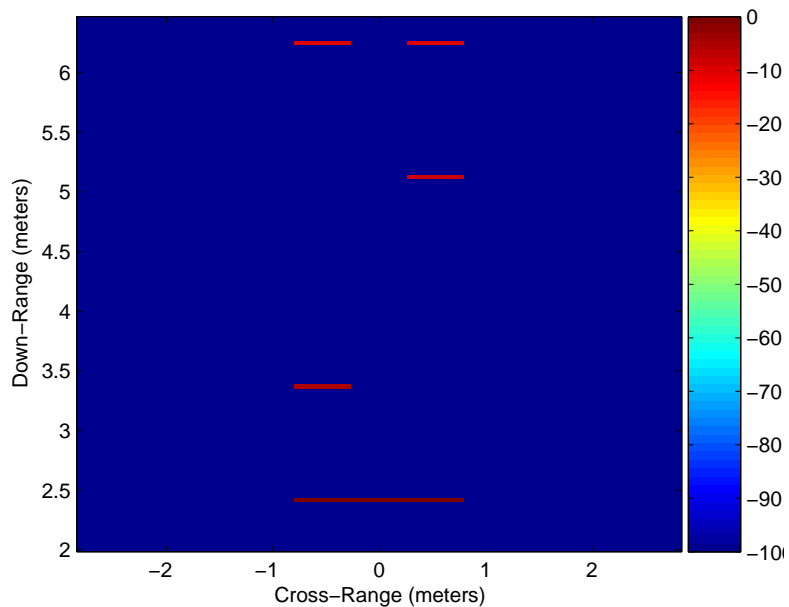

(a)

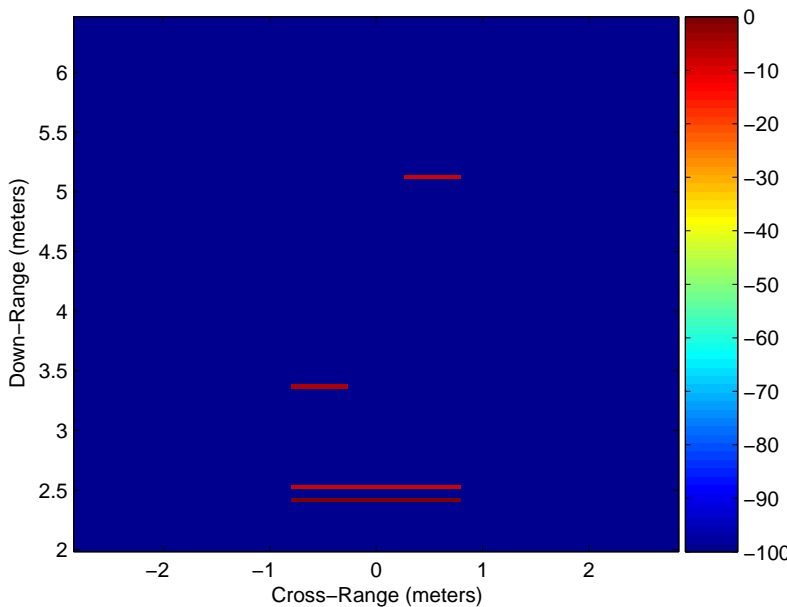

(b)

Fig. 16. Performance of the proposed approach considering the reverberation effect of the front wall: (a) Solid concrete wall (attenuation of $30 \mathrm{~dB}$ ), (b) Adobe brick wall (attenuation of $15 \mathrm{~dB}$ ). 


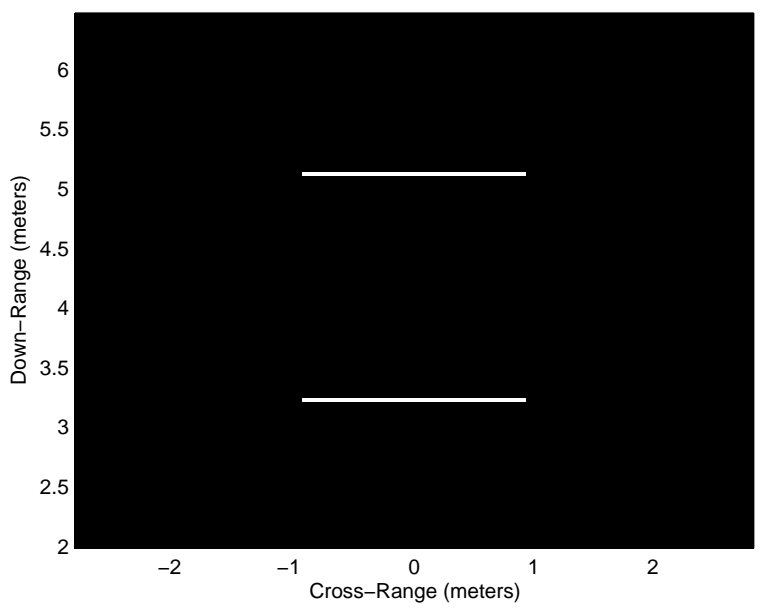

Fig. 17. Geometry of the lab scene

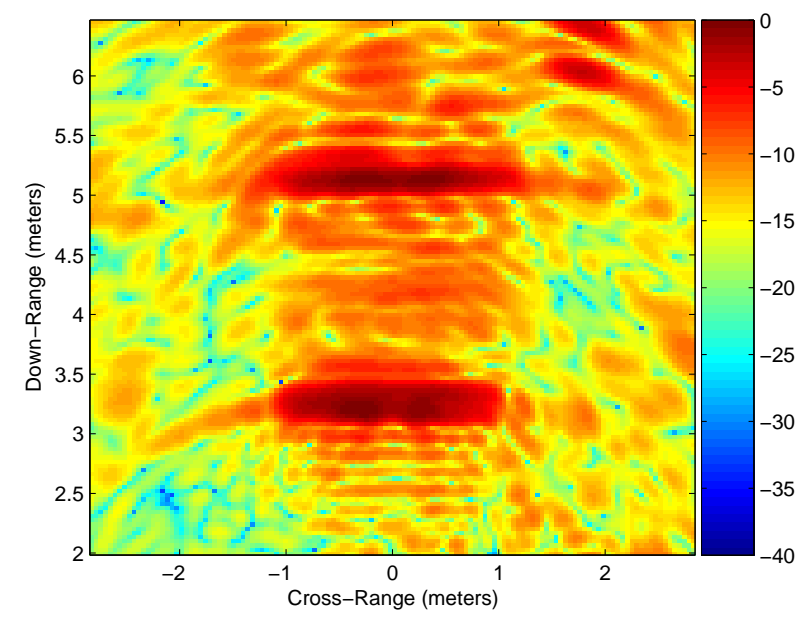

Fig. 18. Through-the-wall radar image using conventional backprojection with $100 \%$ of the data volume. 


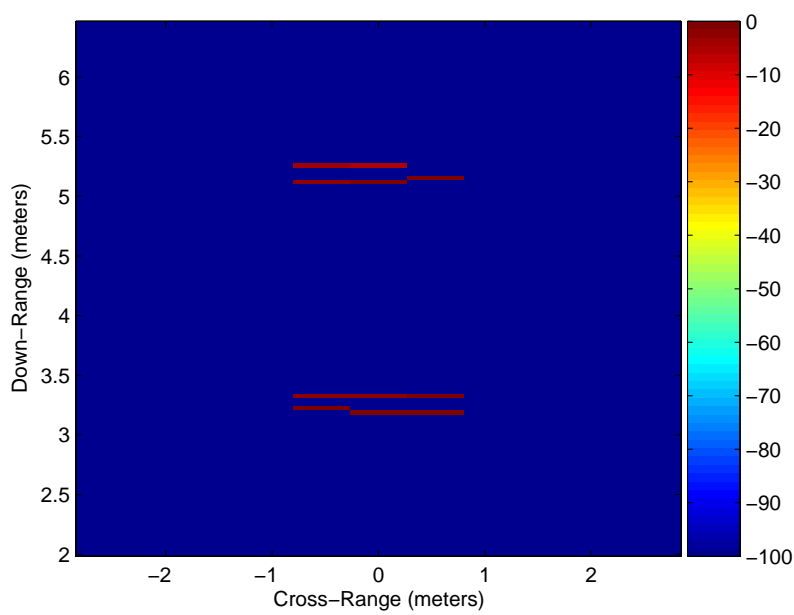

Fig. 19. Reconstructed image from the recovered sparse vector with the proposed approach using OMP and $6.4 \%$ data.

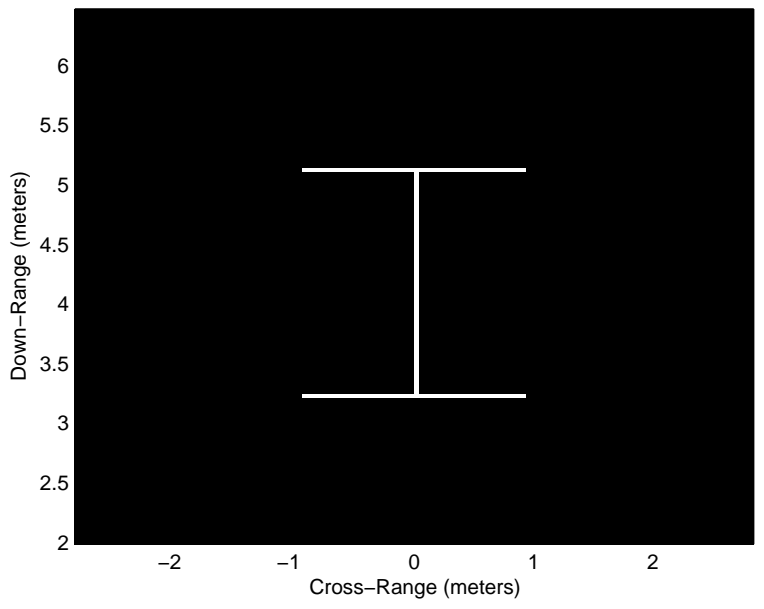

Fig. 20. Geometry of the lab scene 


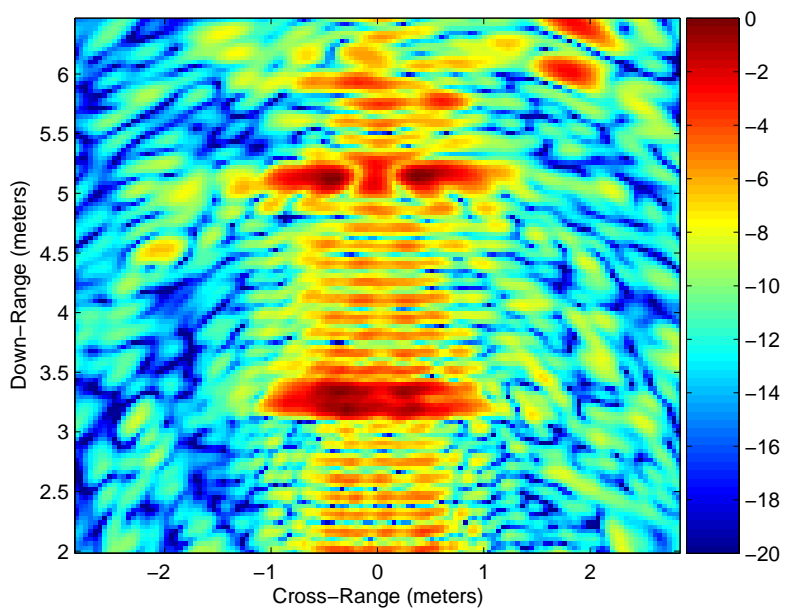

Fig. 21. Geometry of the lab scene

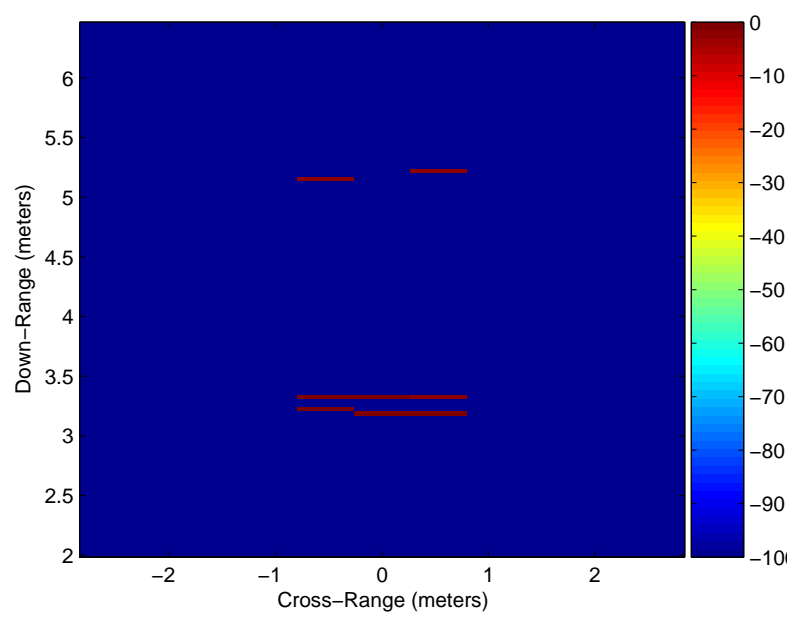

(a)

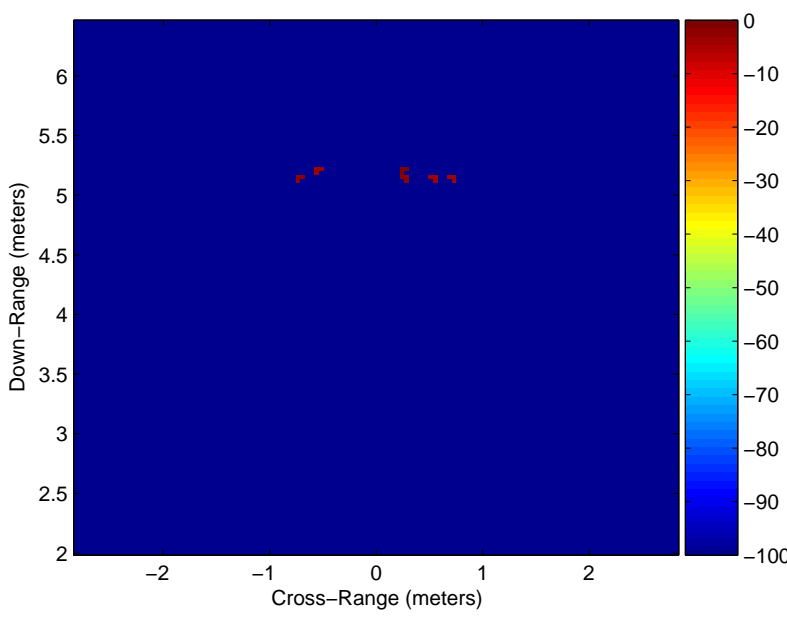

(b)

Fig. 22. Reconstructed image from the recovered sparse vector using OMP and $6.4 \%$ data: (a) Proposed approach for wall detection, (b) Proposed approach for corner detection. 